\title{
La presse francophone grecque de la première moitié du $\mathrm{Xx}^{\mathrm{e}}$ siècle
}

The French-speaking Greek press of the first half of the $20^{\text {th }}$ century

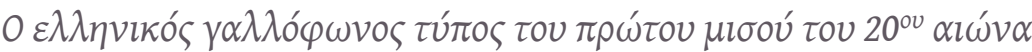

\section{Despina Provata}

\section{OpenEdition}

\section{Journals}

Édition électronique

URL : https://journals.openedition.org/ceb/16053

DOI : $10.4000 /$ ceb. 16053

ISSN : 2261-4184

Éditeur

INALCO

Édition imprimée

ISBN : 9782858313693

ISSN : 0290-7402

\section{Référence électronique}

Despina Provata, "La presse francophone grecque de la première moitié du xxe siècle », Cahiers

balkaniques [En ligne], 47 | 2020, mis en ligne le 21 août 2020, consulté le 06 juillet 2021. URL : http:// journals.openedition.org/ceb/16053; DOI : https://doi.org/10.4000/ceb.16053

\section{(c) (7) (8)}

Cahiers balkaniques est mis à disposition selon les termes de la Licence Creative Commons Attribution - Pas d'Utilisation Commerciale 4.0 International. 


\title{
La presse francophone grecque de la première moitié du $\mathrm{Xx}^{\mathrm{e}}$ siècle
}

\author{
The French-speaking Greek press \\ of the first half of the $20^{\text {th }}$ century

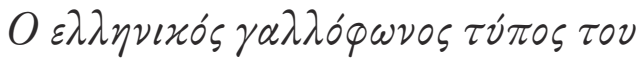

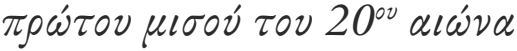

Despina Provata

Université nationale et capodistrienne d'Athènes

\section{Introduction}

La presse francophone grecque naît au début du $\mathrm{XIX}^{\mathrm{e}}$ siècle pour décrire une trajectoire remarquable avant de s'éteindre dans la seconde moitié du $\mathrm{Xx}^{\mathrm{e}}$ siècle. Initialement associée à la présence d'une diaspora française et européenne sur le sol hellène depuis les années de l'Insurrection grecque, elle est aussi liée à l'histoire de la langue française en Grèce et au choix délibéré des élites locales de s'approprier cette langue et de l'utiliser dans la sphère privée, comme dans la sphère publique. Si le français réussit effectivement à s'implanter dans le pays au point de voir naître un lectorat francophone à long terme, c'est également parce qu'il devient matière obligatoire dans l'enseignement grec à partir de $1836^{1}$. On assiste donc à la mise en place progressive d'une communauté de lecteurs qui prend la France pour modèle civilisationnel. C'est dans ce cadre particulier d'un pays où la langue française n'a jamais été imposée, ni comme langue officielle, ni comme langue seconde, comme cela a été le cas dans d'autres pays méditerranéens à forte tradition francophone ${ }^{2}$,

1. Provata, 2011, p. 181-192.

2. Kraemer, 2001. 
que voit le jour une presse originale, publiée soit dans la seule langue française, soit en présentation bilingue, français-grec ${ }^{3}$.

Ma contribution à ce stade de la recherche ne consiste pas à retracer en quelques pages toute l'histoire de la presse francophone grecque de la première moitié du $\mathrm{XX}^{\mathrm{e}}$ siècle. Je m'efforcerai dans ce premier temps d'établir un inventaire aussi raisonné que possible, afin d'en dégager les lignes de force qui, elles, permettront d'affiner sa périodisation, de dessiner sa typologie et de recenser les initiateurs de cette production éditoriale particulière. Une première cartographie de ce déploiement va nécessairement refléter le cadre spatio-temporel au sein duquel ces publications ont vu le jour au cours du $\mathrm{Xx}^{\mathrm{e}}$ siècle.

\section{Repérage, inventaire et cartographie}

Mais avant tout, il faudrait définir l'aire recouverte par l'expression de « presse hellénique en langue française ». En effet, pour un pays dont les frontières ne cessent de se modifier depuis sa fondation en 1830 jusqu'à la seconde moitié $\mathrm{du} \mathrm{xx}^{\mathrm{e}}$ siècle, il convient d'entendre le terme de « presse hellénique » moins en référence à la surface officielle de l'État qu'à son aire d'influence politique et culturelle. Ainsi, par « presse francophone grecque » je désigne les feuilles partiellement ou entièrement rédigées en langue française, par des Grecs - ou parfois des étrangers - quel qu'en soit le lieu d'édition, mais qui sont les porteparoles des aspirations politiques et culturelles des Grecs. J'ai donc inclus dans ce corpus, en premier lieu, les périodiques publiés en Grèce, journaux ou revues à périodicité plus ou moins régulière. En second lieu, les périodiques publiés à l'étranger en français par des Grecs qui s'engagent pour la défense de l'hellénisme ou pour la promotion de la culture grecque 4 . Pour ces deux catégories confondues, j'ai pour l'instant répertorié près de 40 titres paraissant au cours des premières décennies $\mathrm{du} \mathrm{Xx}^{\mathrm{e}}$ siècle. Pour la constitution de ce corpus, j'ai dans un premier temps utilisé deux sources précieuses, mais malheureusement incomplètes :

3. Ma recherche sur la presse francophone est loin d'être achevée, mais j'envisage de constituer un répertoire complet de ces périodiques. À ce jour, alors que la recherche reste encore ouverte, une centaine de titres sont répertoriés pour les $\mathrm{XIX}^{\mathrm{e}}$ et $\mathrm{XX}^{\mathrm{e}}$ siècles. Pour une première cartographie du phénomène, consulter Provata, 2017, p. 281-296.

4. J'ai laissé de côté les revues savantes, liées au développement des études néo-helléniques en France et qui soit sont des organes des associations scientifiques, comme La Revue des Études grecques (1888-1928), publication trimestrielle de l'Association pour l'encouragement des études grecques en France, soit sont associées aux chaires universitaires néo-helléniques en France, telle la revue des Études néo-helléniques publiée par Octave Merlier (1868-1976). Voir à ce sujet Arnoux-Farnoux, 2015, p. 186-193. 
l'inventaire de Christopoulos (1994) qui recense la collection de la Bibliothèque du Parlement, la collection la plus complète de journaux et revues en Grèce, et

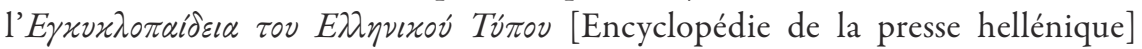
(2008). Cependant, de nombreux titres restaient encore non répertoriés, surtout ceux qui avaient paru à l'étranger. Rassembler ce corpus n'est pas une tâche aisée, car ces feuilles sont non seulement dispersées dans des bibliothèques très diverses, mais également victimes des outrages du temps, de sorte que leurs séries sont bien souvent incomplètes ou font tout simplement défaut. Parfois, de surcroît, nous ne disposons que de quelques numéros.

Pour ce qui est des journaux francophones publiés à Athènes, la plupart sont des parutions hebdomadaires, peu de feuilles existant sous forme de quotidien. Au travers de leurs éditoriaux et de leurs articles de fond se dégage leur ligne programmatrice, à savoir l'information de l'opinion publique aussi bien sur les faits de politique intérieure que sur les questions d'intérêt européen ou international, des plus importants aux plus triviaux. Citons à titre d'exemple Le Progrès (1898-1910), Le Courrier d'Orient (1903-1905) et notamment Le Messager d'Athènes (1875-1980 [?]), journal emblématique qui connaît la plus grande longévité de toute la presse francophone grecque 5 . Et parmi ces titres, une seule revue littéraire, L'Hellénisme contemporain (1935-1941, 1947-1956). Pendant l'occupation allemande (1941-1945), et à l'instar de la quasi-totalité des feuilles hellénophones, les journaux et revues francophones suspendent leur publication. Après la Seconde Guerre mondiale, seuls Le Messager d'Athènes et L'Hellénisme contemporain reprennent leur activité, tandis que paraît à la Libération pour une brève période d'un an seulement l'Écho de France (1945-1946) qui se veut « journal politique indépendant ».

Si la plupart des journaux grecs en langue française ont leur siège sur le territoire de l'État grec, on ne peut pas se faire une idée d'ensemble de cette presse particulière sans se pencher sur les périodiques que publiaient les communautés grecques à l'étranger, au premier rang desquels on compte ceux de Paris.

En France, et notamment à Paris, se constitue en effet à partir de la fin du $\mathrm{XIX}^{\mathrm{e}}$ siècle, une importante communauté grecque qui s'étoffe de façon spectaculaire de 1916à $1931^{6}$. Composée initialement d'une bourgeoisie d'affaires et d'intellectuels, elle accueille aussi en ses rangs des artisans, des commerçants et des professions libérales. Cette communauté va connaître un essor considérable

5. Sur Le Messager d'Athènes, voir l'article de Joëlle Dalègre « Le Messager d'Athènes, ou la défense de l'Hellénisme » dans le présent volume.

6. Sur la construction de la communauté grecque à Paris voir Papadopoulou, 2004 ; Manitakis, 2000. 
pendant l'entre-deux-guerres lorsqu'aux Grecs déjà installés en France s'ajoutent, en 1915, d'importants effectifs d'ouvriers destinés aux industries d'armement. Ainsi, à la veille de la Seconde Guerre mondiale, la France possède la collectivité hellénique la plus nombreuse d'Europe occidentale, soit 19123 Grecs en $1931^{7}$. À Paris, deuxième ville d'accueil des Grecs en France, sont publiés en français, parmi d'autres titres, L'Hellénisme (1904-1912), Graecia (1910-1914), L'Hellénisme irrédimé (1919), Le Journal des Hellènes (1916-1931), Les Études franco-grecques (1918-1921), La Méditerranée orientale (1917-1919). Toutes ces feuilles partageaient un objectif commun : cultiver les relations franco-helléniques, attirer l'attention de la communauté française et européenne sur les questions politiques du moment et surtout, à un moment où la Grèce était discréditée après la honteuse défaite lors de la guerre gréco-turque de 1897, aider le pays à retrouver son prestige perdu. Enfin, cette presse se faisait également l'écho des progrès effectués dans le domaine de l'économie, de la littérature et des arts.

\section{Quel public-cible pour cette presse?}

Évaluer le public auquel s'adressent les journaux de langue française s'avère une tâche difficile. Nous ne disposons d'aucun indice sur les tirages et n'avons que très peu d'indications sur la diffusion géographique de ces feuilles. Nous pouvons donc seulement procéder à des déductions nécessairement approximatives d'ordre social pour définir les milieux potentiels de lecteurs de ces périodiques. Comme ce fut le cas au cours du $\mathrm{XIX}^{\mathrm{e}}$ siècle, certaines de ces feuilles ont un double ciblage : informer d'une part le public français et/ou européen résidant dans le pays et à l'étranger, mais aussi répondre à l'attente d'une partie du lectorat grec pour qui l'utilisation de la langue française était un signe de distinction et d'appartenance sociale, à savoir la bourgeoisie cultivée et les gens d'affaires.

Cela nous conduit à nous pencher sur le deuxième ensemble de notre corpus, celui des journaux bilingues. À Athènes, on l'a dit, sont publiés de nombreux journaux français-grec, où la langue française occupe une place à proportion variable, dont la fluctuation, obéit à des critères très variés. Dans certains cas, le texte français figure en regard du texte grec et en constitue une traduction plus ou moins fidèle, tel est le cas du Progrès qui paraît au tournant du siècle et de son successeur Le Progrès d'Athènes (1918-1926) pour n'en citer que deux. Dans d'autres cas, qui ne se limitent pas à la capitale de l'État grec, le contenu des articles français d'un journal ne coïncide pas avec ceux qui sont rédigés en grec : Le Courrier d'Orient, par exemple, opte pour une double édition, en français et

7. Bruneau, 1996, p. 489-490. 
en grec, éditions parallèles et différentes ${ }^{8}$. La place du français dans une feuille peut aussi être très limitée et obéir à des raisons particulières. Citons l'exemple du journal hellénophone $\Delta \omega \delta \varepsilon x \dot{\alpha} \nu \eta \sigma o \zeta / L e$ Dodécanèse qui circule en Alexandrie durant l'entre-deux-guerres (1925-1932, 1934-1937), là où avait été transféré le centre de la lutte pour la libération des îles et pour leur annexion à l'État grec ; ce journal publie toujours son éditorial dans les deux langues, alors que le reste de ses articles est exclusivement rédigé en grec. On peut également se trouver en présence d'une utilisation occasionnelle du français. C'est notamment le cas du journal hellénophone 'E $E \varepsilon \sigma \sigma \alpha(1919)$ - dont je n'ai pu à ce jour repérer qu'un seul numéro - mais qui constitue un indicateur du rôle particulier qu'il entendait jouer à ce moment précis. En effet, le débarquement de l'armée d'Orient dans la région de Salonique ne laisse pas ses rédacteurs indifférents. Ceux-ci, reconnaissant sans doute un besoin d'information des soldats français expatriés, publient une partie de leurs contenus en français, et même un poème en langue française ${ }^{9}$. Dans d'autres cas, enfin, nous sommes en présence d'un usage exclusif de la langue française, comme dans le cas du Messager d'Athènes ou de L'Hellénisme Contemporain.

\section{Presse et identité nationale}

Née pendant les années de l'Insurrection, la presse hellénique de langue française a d'emblée été soucieuse des enjeux nationaux, principe défendu sans faillir tout au long du $\mathrm{Xx}^{\mathrm{e}}$ siècle. Il suffit déjà, pour s'en rendre compte, de parcourir les titres des périodiques du $\mathrm{xx}^{\mathrm{e}}$ siècle : L'Hellénisme (1904-1912), Le Monde Hellénique (1906-1910), L'Hellénisme irrédimé (1919), Le Patriote (1906-1907), L'Hellénisme Contemporain ou Le Journal des Hellènes (1922-1930). Ces titres éloquents constituent un échantillon de ces feuilles qui exprimaient l'identité grecque. D'autres, tout en s'adressant à la totalité du public grec francophone, misent sur la représentation d'Athènes, nouvelle capitale du pays et lieu de l'action politique et culturelle de la Grèce moderne : Le Progrès d'Athènes, Le Journal d'Athènes (1923-1925), Le Messager d'Athènes.

Presse donc naturellement « nationale », la presse francophone grecque joue un rôle de premier plan dans l'affirmation de la nation et, par suite, dans la construction du sentiment national. En effet, l'histoire de cette presse continue de

8. Panagou, 2017, p. 275-280.

9. $E \delta \varepsilon \sigma \sigma \alpha, 1^{\text {er }}$ mars $1919,1^{\text {ère }}$ année, $n^{\circ} 5$, p. 3 . « Je ne saurai cependant affirmer si cette pratique s'est poursuivie ni quelle fut la longévité de cette feuille, d'ailleurs concurrencée par un grand nombre de publications qui paraissent à Salonique lors de la Grande Guerre afin de servir les buts de la propagande de l'Entente », Flitouris, 2017, p. 260-261. 
s'articuler au $\mathrm{XX}^{\mathrm{e}}$ siècle sur les événements qui ont secoué le pays. Cette particularité explique sans doute le fait que certaines périodes sont plus fécondes que d'autres. Telle serait par exemple le début de la lutte en Macédoine (1904-1908), qui a vu une recrudescence de nouveaux journaux.

Entre 1904 et 1908, lorsque la Macédoine, s'enflamme en un « champ clos » d'une rivalité acharnée entre les Grecs, les Serbes et les Bulgares ${ }^{10}$, des associations patriotiques visant à soutenir le combat des populations macédoniennes face au danger bulgare sont fondées à Athènes ; elles organisent protestations et manifestations et exercent une pression sur le gouvernement grec qui ne voulait pas s'engager trop ouvertement dans la lutte armée. Parmi ces sociétés, deux des plus actives, « l'Association centrale Macédonienne » et l'association « Hellénisme » encouragent la publication de feuilles en langue française afin d'informer les lecteurs français et européens, et de sensibiliser l'opinion européenne dans le but d'obtenir un soutien politique pour la Grèce.

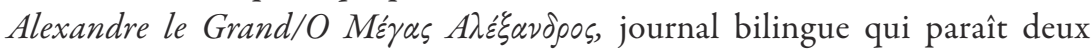
fois par semaine entre 1905 et 1907 à Athènes, est l'organe de l'Association

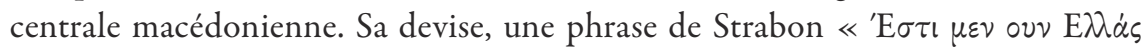

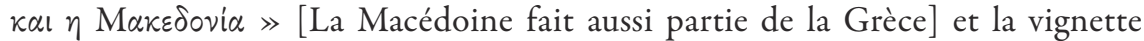
qui l'accompagne dans le bandeau du journal (une tête d'Alexandre le Grand), ne laissent aucun doute sur l'objectif de cette publication. Pour qui ne l'aurait pas encore compris, il est d'ailleurs énoncé de la manière la plus claire dans sa profession de foi initiale : « rendre la Macédoine tout entière à l'Hellénisme ». Dirigé par Th. N. Synodinos, le journal consacre son contenu exclusivement à la question macédonienne. En adoptant un discours de polémique rigoureuse contre l'ennemi, il s'emploie à informer ses lecteurs sur les cruautés commises par les Bulgares et sur le rôle de la diplomatie européenne. Ses intentions sont clairement énoncées :

[Il s'agit] d'encourager de toutes les manières nos compatriotes à lutter vaillamment pour l'idée nationale, d'éclairer l'opinion publique aussi bien chez nous qu'en Europe sur les questions en litige ainsi que sur les complications qui surgissent en tout instant en Macédoine et de faire enfin connaître au monde civilisé tout entier toutes les sauvageries de nos ennemis en Macédoine et toutes les intrigues diplomatiques des divers Représentants ou Agents des Grandes Puissances [qui] tendent à empêcher le rétablissement national des Macédoniens. [...] faire de notre feuille un projecteur 
lumineux capable de jeter une pleine lumière sur la question de Macédoine qui fera découvrir tout ce que qui se cache sous les plis tortueux de la diplomatie et sous la capote du Bulgare fourbe et $\operatorname{assassin}^{11}$.

Outre la présentation des faits de la guerre, le journal consacre une grande partie de son contenu à l'histoire de la Macédoine, depuis l'Antiquité jusqu'aux temps modernes. Il critique la diplomatie européenne qui hésite à intervenir et, afin de ranimer le sentiment patriotique, publie les biographies des héros tombés sur le champ de bataille. La première, consacrée à Paul Mélas, est accompagnée d'un portrait photographique du héros ${ }^{12}$. C'est qu'à partir du quatrième numéro, le journal devient illustré, la photographie se mettant ainsi au service du récit de guerre. Il s'agit non seulement d'immortaliser la figure du héros tué au combat, mais également de construire une information visuelle pour présenter d'une manière aussi réaliste que possible les corps de ceux qui ont enduré le martyre sous la main bulgare et les actes de violence injustifiables des comitadjis. Bien que la totalité du contenu de ce journal soit dédiée à la grande cause de l'hellénisme, il accorde une place à l'expression littéraire ; mais là encore, il s'agit de poèmes inspirés de la même thématique.

La société « L’Hellénismos », fondée en 1892 par Georges Krémos ${ }^{13}$ et dirigée à partir de 1894 par Néoclis Kazazis ${ }^{14}$, ne laisse aucun doute sur ses objectifs et son caractère patriotique. Elle défend sans faillir la Grande Idée comme destin national et travaille à renforcer le sentiment patriotique en cultivant les aspirations irrédentistes des Grecs. Cette société qui publiait déjà depuis 1898

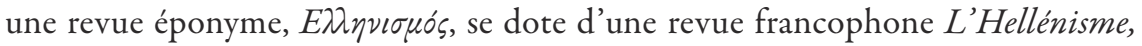
publiée entre 1904 et 1912 à Paris, et d'un journal franco-hellénique intitulé

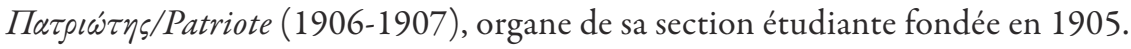

L'Hellénisme (1904-1912), avec son homologue athénien, va orchestrer la conquête des esprits européens. La revue française se veut « le moyen par lequel la voix grecque se fera entendre dans les centres européens, mais aussi le moyen de communication avec la presse européenne ». La société s'efforce par ailleurs de raviver le mouvement philhellène en France et crée la Ligue pour la défense des droits de l'Hellénisme dont l'objectif était de défendre les intérêts des Hellènes

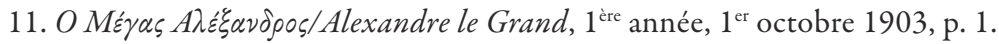

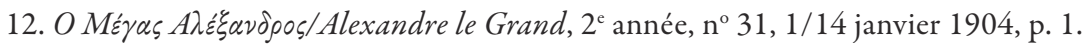

13. Georges Krémos (1839-1926), écrivain, professeur et historien.

14. Néoclis Kazazis (1849-1936), écrivain, professeur de droit et recteur de l'université d'Athènes (1902-1903). 
vivant dans les régions encore soumises à l'Empire ottoman. Autour de la revue et de la ligue gravite un réseau d'intellectuels français, tels l'homme politique Denys Cochin, le baron Pierre de Coubertin, le publiciste Alfred Berl, l'écrivain Maurice Barrès ou l'archéologue Georges Perrot qui signent plusieurs de ses pages.

Le Patriote, organe officiel des étudiants hellènes, adopte un discours similaire au sujet des revendications nationales et choisit comme langues de publication le grec et le français (une partie des contenus, celle concernant la lutte en Macédoine, est publiée dans les deux langues, le reste uniquement en grec). Dans sa profession de foi initiale, le directeur du journal, P. S. Apostolopoulos, étudiant en droit, explique l'une des raisons de la publication du journal ; l'ennemi bulgare, écrit-il :

[...] a combiné l'œuvre du poignard assassin et des bombes - ses moyens d'agir - avec des imprimés divers amplement et à titre gracieux distribués en Europe dont le but principal n'est que d'altérer la réalité et en un seul mot d'empoisonner et de surprendre l'opinion publique universelle ${ }^{15}$.

Bien qu' Alexandre le Grand et Le Patriote, étant des journaux bilingues publiés en Grèce, soient en principe destinés à un lectorat grec francophone, ils sont aussi des organes de propagande. Cela explique qu'ils ne négligent pas l'importance que peut avoir leur lectorat étranger pour la promotion des revendications nationales. D'où leur choix de servir gratuitement des exemplaires à l'étranger. Alexandre le Grand « est envoyé à titre gratuit aux diplomates, aux hommes politiques éminents, aux ethnologues et aux sociétés de géographie ainsi qu'aux principaux organes du monde entier », comme il est signalé au bandeau du journal. Quant au Patriote, il nous livre des données quantitatives : 4000 exemplaires en 1906 et 5000 à partir de 1907 sont destinés à être distribués gratuitement à l'étranger.

Mais, même lorsque les questions nationales ne sont pas l'unique objectif ou la priorité d'un journal, elles y occupent une place importance pendant cette période troublée. Tel est le cas du Monde Hellénique, journal entièrement rédigé, lui, en langue française, qui voit le jour à Athènes entre 1906 et 1910. Ses éditeurs, Spyridon Pappas, spécialiste en histoire économique et Jean Dargos (pseudonyme d'Elbert Jacques Pierre de Hochepied) critique et traducteur, tiennent un rôle complémentaire dans le journal : alors que le premier signe les articles politiques, le second couvre le domaine culturel. Le Monde bellénique, qui devient bilingue à partir de 1909, opte pour la neutralité. Face aux organes de propagande patriotique, tels Alexandre le Grand et Le Patriote qui paraissent au même moment, il tente d'adopter un discours communicationnel susceptible 
de ne trahir aucune coloration politique, sans toutefois parvenir à cacher sa préférence monarchique. Journal d'information en premier lieu, il accorde une place importante aux questions politiques qui occupent l'actualité, notamment l'avenir de la Macédoine et celui de la Crète. Or, tout en soutenant les aspirations irrédentistes des Hellènes, Le Monde hellénique souscrit aussi à la médiatisation de la culture hellénique à l'étranger. À travers ses colonnes seront présentées toutes les activités culturelles du pays : nouvelles archéologiques, activité théâtrale de la capitale, parutions littéraires ou scientifiques, tout en donnant une place particulière à la traduction de la littérature néo-hellénique ${ }^{16}$.

Mais si la presse francophone sert d'appui aux revendications nationales, elle peut également devenir organe de propagande entre les mains de forces étrangères. Ainsi, durant la Grande Guerre, le recours à la langue française a même pu faire écran à des endoctrinements allemands. En 1915, les rapports se détériorent entre le roi Constantin, germanophile, et son Premier ministre Elefthérios Venizélos, qui s'allie, lui, à la Triple Entente, et conduisent à un affrontement qui aboutira, en 1916, au débarquement des forces britanniques et françaises au Pirée. Dans ce contexte, on ne saurait s'étonner que, dès 1915 , la propagande allemande se soit organisée en finançant un journal de langue française, La Gazette d'Athènes. Cette dernière, visant le lectorat francophone, étale son hostilité face à l'Entente et à la politique menée par Venizélos ${ }^{17}$.

Jusque-là, la France qui jouissait d'un certain état de grâce dans un pays nettement francophile n'avait pas senti le besoin de réagir. Elle se contentait de diffuser des communiqués de presse. Mais avec l'arrivée de Gustave Fougères à la tête de l'École française d'Athènes, qu'il dirigera de 1913 à 1919, une propagande en faveur de la France s'organise pour répondre à l'action menée par l'Allemagne. Officiellement investi de la direction de la propagande en Grèce, Fougères profite de la collaboration de Venizélos et de Basil Zaharoff ${ }^{18}$ pour mettre en marche une subtile machine de promotion des intérêts français. La création de l'agence Radio, début 1916, permettait à la légation de France de diffuser aux journaux grecs, et surtout aux organes vénizélistes, des nouvelles soigneusement contrôlées, inédites, ainsi que des interviews. La France apporte maintenant elle aussi, une aide financière aux journaux hellénophones favorables à l'Entente, et décide à partir d'avril 1916 d'étendre son champ d'action avec la création d'une feuille en français, Le Progrès d'Athènes. Organe officieux de la légation, il paraît de 1918

16. Arnoux-Farnoux, 2017, p. 186-193.

17. Montant, 1989, p. 63-63.

18. Basil Zaharoff (1849-1836) homme d'affaires et mécène, originaire d'Anatolie et proche de Venizélos. 
à 1926, d'abord deux fois par semaine, puis devient quotidien. Enfin, en 1917 aurait paru, à l' instigation de la propagande française, un hebdomadaire de langue française, La Vérité, dont je n'ai pu à ce jour trouver aucune trace ${ }^{19}$.

Pendant cette même période, de la Grande Guerre et de l'entre-deux-guerres, l'hellénisme bénéficie du soutien des périodiques publiés à Paris : L'Hellénisme irrédimé (1919), Le Journal des Hellènes (1916-1930), La Méditerranée orientale (1917-1919) ou Les Études franco-grecques (1918-1921) se chargent de la défense de l'hellénisme et de la promotion des intérêts du pays. Le Journal des Hellènes, qui paraît initialement à Genève avant de transférer son lieu de publication à Paris, se qualifie comme « organe panhellénique national pour la défense des intérêts grecs paraissant à Paris ». Destiné aux colonies helléniques de l'Europe centrale et occidentale, c'est l'un des journaux les plus riches en contenu et le plus dynamiques de la communauté grecque de Paris. Son fondateur, Luc Pyrrhus, qui souhaitait contribuer au développement des relations franco-helléniques et attirer l'attention du public européen sur les progrès grecs réalisés dans le domaine de la politique, des arts et de l'économie, choisit pour devise « faire aimer la Grèce à l'étranger ». Bien que francophone, le journal n'hésitera pas, en signe de reconnaissance envers la Grande-Bretagne pour son soutien lors de la guerre, à adopter provisoirement la langue anglaise aussi. Le Journal des Hellènes opte pour la neutralité politique et alimente ses colonnes de nouvelles concernant la politique et la diplomatie grecques et internationales, puisées souvent dans les grands quotidiens européens. Organe de la communauté grecque de Paris, ses pages constituent une source précieuse pour les activités de celle-ci. D’autre part, les colonnes du journal prennent soin d'apporter aux lecteurs des nouvelles de Grèce qui servent non seulement à informer, mais aussi à entretenir les liens affectifs des Grecs expatriés avec la mère-patrie ${ }^{20}$.

Comme on l'a vu, la presse francophone publiée sur sol grec a été pour la France une arme de propagande. Après la guerre, lorsque la presse et l'opinion publique françaises, séduites par le nouveau visage de la Turquie, se détournent de la Grèce, le gouvernement grec cherchera des appuis à l'étranger. Léon Maccas ${ }^{21}$, proche de Venizélos, comme des milieux diplomatiques français à Athènes, publie la revue mensuelle Études franco-grecques (1918-1921) en vue, écrit-il, de « consolider et de resserrer les liens politiques, économiques et intellectuels

19. Montant, 1989 , p. $70-77$.

20. Korma, 2008, p. 428-430.

21. Léon Maccas (1892-1972) haut-fonctionnaire, diplomate, homme politique et homme d'État grec, a collaboré à des organes de presse français et est l'auteur de plusieurs ouvrages historiques. 
qu'une longue tradition, l'intérêt commun et la ressemblance de leurs aspirations ont noués autour de la France et de la Grèce ${ }^{22} \gg$. Son entreprise, financée par le gouvernement grec, profitera de la collaboration de personnalités françaises éminentes, parmi lesquelles on retrouve Gustave Fougères et Maurice Barrès, ainsi que Théodore Homolle, René Puaux et Joseph Reinach, tous membres de l'École française d'Athènes ${ }^{23}$.

\section{Les revues francophones : la promotion de la vie littéraire et culturelle}

Comme cela a déjà été mentionné, les journaux francophones sont davantage orientés vers la défense des intérêts de la Nation, c'est pourquoi ils laissent finalement peu de place à la vie culturelle et littéraire. Mais lorsqu'ils y consacrent leurs colonnes, c'est toujours en obéissant au même objectif : forger une image positive de leur pays, vanter ses conquêtes dans le domaine des lettres et des arts, prouver aux lecteurs européens que la Grèce mérite à titre égal une place parmi les autres nations. Si la presse francophone grecque est nationale par ses contenus, elle l'est donc également par les lectures qu'elle propose. Car participer au destin commun du peuple signifie aussi participer à la construction de la littérature nationale. Ce rôle est surtout dévolu aux deux revues littéraires francophones qui circulent dans la première moitié du $\mathrm{xx}^{\mathrm{e}}$ siècle : Graecia à Paris et L'Hellénisme contemporain à Athènes.

Publiée à Paris sous le patronage littéraire de Juliette Adam, grande amie de la Grèce ${ }^{24}$, Graecia est l'organe du monde grec et philhellène. Deux comités, l'un à Paris et l'autre à Athènes, composés de personnalités grecques et françaises soutiennent cette initiative et collaborent à ses pages. Revue de littérature et d'art, Graecia offre à ses lecteurs « une synthèse vivante et littéraire de la Grèce moderne »; en outre, elle prend soin de diffuser dans le monde grec l'actualité littéraire, théâtrale et artistique de France, devenant ainsi une passerelle culturelle entre les deux pays ${ }^{25}$.

C'est un fait que Graecia n'omet aucun aspect de la Grèce moderne : elle s'ouvre à la littérature néohellénique qu'elle publie en traduction, elle accueille dans ses pages plusieurs articles et études portant sur les écrivains grecs, ou suit régulièrement le conflit linguistique qui agite la vie littéraire en Grèce et oppose

22. « Notre but », Études franco-grecques, $1^{\text {ère }}$ année, $\mathrm{n}^{\circ} 1$, avril 1918.

23. Kitsikis, 1963, p. 232, 226-227 ; Eglezou, 2009, p. 29.

24. Provata, 2007, p. 63-76.

25. Sur le rôle des revues dans les transferts culturels, voir Loué, 2005, p. 195-208. 
les partisans de la langue pure, « la katharévoussa », aux partisans de la langue populaire, « le démotique ${ }^{26} \gg . S^{\prime}$ inspirant du modèle de la revue généraliste, elle se penche également sur les progrès réalisés dans le domaine de l'économie et du commerce, voire des infrastructures du pays ; elle souligne à chaque occasion la transformation de la société hellénique qui, elle aussi, s'est engagée dans la voie de la modernisation. Organe d'information du monde grec, Graecia s' intéresse aussi à l'actualité française, hellénique et mondiale et circule avec un supplément intitulé «Graecia-Journal » dans lequel sont publiés les actualités de la vie parisienne et celles des communautés grecques à l'étranger, les chroniques financières, des articles sur la mode et la vie mondaine des deux pays, des renseignements et informations divers, des annonces publicitaires. Enfin, comme Graecia circule à un moment où s'exacerbe la lutte armée dans les Balkans, elle ne manque pas de proposer à ses lecteurs des analyses rigoureuses sur ces questions politiques du moment.

Pendant l'entre-deux-guerres, en 1937, voit le jour à Athènes l'Hellénisme Contemporain dont la publication, interrompue pendant l'occupation allemande, reprend en 1947 et se poursuit jusqu'en 1956. Fondée et dirigée par une Française, Henriette Avatanghélos, la revue s'adresse à un public savant, français et international. Son objectif était de promouvoir la vie littéraire et culturelle grecque à l'étranger, engagement qui bénéficiait du soutien de l'État grec, puisque la revue sortait des presses officielles de l'État ${ }^{27}$. À travers les traductions littéraires, nombreuses dans la première période de sa parution, ou la publication d'études scientifiques, aussi bien que par les articles réguliers qui couvrent toute l'activité culturelle, cette revue contribue à redynamiser la vie culturelle grecque. Son orientation littéraire est en grande partie définie jusqu'en 1944 - date de sa mort - par Tellos Agras ${ }^{28}$, principal collaborateur d'Avatanghélos. Il sera par la suite remplacé par Dionyssios Zakynthinos, professeur d'histoire byzantine, qui prend le relais aux côtés d'Avatanghelos avant de prendre la direction de la revue à la mort de son éditrice, en 1955. On ne saurait donc s'étonner que, pendant la décennie 1947-1956, la place de la littérature se trouve réduite au profit des articles portant sur l'histoire byzantine et néo-hellénique, sur le folklore, la critique littéraire et les comptes rendus de livres.

26. Milioni, 2009, p. 111-124.

27. Yiakovaki, 2008, p. 419-421.

28. Tellos Agras (1899-1944), pseudonyme de Evangelos Ioannou, poète et critique qui collabora à plusieurs revues de l'entre-deux-guerres. 


\section{Presse économique spécialisée}

Mais le français n'est pas seulement une langue de culture, de savoir et d'information. C'est aussi la langue alors utilisée dans tout le bassin méditerranéen pour les transactions économiques et commerciales, un instrument d'accès à l'ère de la modernité. Les petites annonces insérées dans la presse proposant des cours de français commercial ou les nombreux manuels de français à usage commercial en circulation dans le pays, certains même destinés à l'autoapprentissage, illustrent cette tendance de la société grecque et attestent le besoin réel du temps de former un personnel spécialisé francophone.

Présents en Grèce depuis la fin du $\mathrm{XIX}^{\mathrm{e}}$ siècle, les journaux économiques spécialisés en langue française ${ }^{29}$ connaissent un essor au $\mathrm{Xx}^{\mathrm{e}}$ siècle, dû au développement du commerce et des grandes compagnies de navigation, de la bourse, ainsi que de la naissance des banques. Certains de ces journaux optent exclusivement pour le français comme Les Annales Franco-belléniques. Politiques, financières, commerciales, industrielles et maritimes (1918-1919), le Journal de la Bourse (1921-1922). D'autres pour le bilinguisme dans le cas de la Bourse d'Athènes/X d'Athènes, L'Économiste/O Oıxovouodóros (1902) et Métochos/L'Actionnaire (1912-1918). Le Bulletin bimensuel de la Chambre de Commerce Hellénique

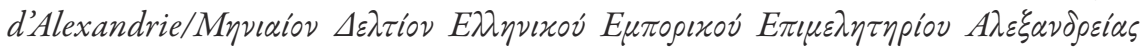
(1902-1962) et le Bulletin de la Chambre de Commerce d'industrie de Volos/ $\Delta \varepsilon \lambda \tau i o v$

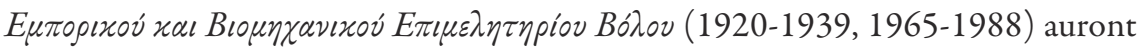
aussi sporadiquement recours à la langue française.

Cette presse, qui témoigne de l'émergence d'une nouvelle catégorie de lecteurs, hommes d'affaires, négociants et rentiers qui font vivre ces feuilles, est en outre liée aux préoccupations de la diplomatie française et aux aspirations économiques de la France dans la région. En effet, en 1917et 1918, la France cherche à regagner la confiance du public grec après les malheureux événements de décembre 1916, marqués par le débarquement des forces alliées au Pirée et le bombardement de la ville d'Athènes. Dans ce cadre, Gustave Fougères, directeur de l'École française d'Athènes de 1916 à 1919 et chargé de la propagande, comme on l'a vu, cherche non seulement à contrebalancer la germanophilie dans le domaine politique et culturel, mais aussi à renforcer la pénétration économique française dans le pays. Ainsi, dans un rapport qu'il rédige en 1917, il propose d'élaborer un plan d'action

29. Deux titres paraissent au cours du $\mathrm{XIX}^{\mathrm{e}}$ siècle: L'Économiste/O Oıxovopohóyos

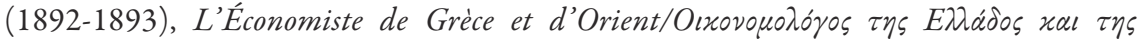

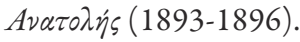


qui, d'une part, ouvrira le marché grec aux produits français en les faisant connaître à la clientèle grecque et, d'autre part, entend informer les milieux commerciaux français des besoins du marché grec ${ }^{30}$. Une recherche plus approfondie des contenus et collaborateurs des journaux économiques pourrait éclairer le rôle que ces feuilles ont joué dans les relations économiques et commerciales bilatérales entre la Grèce et la France. Il convient ici de faire remarquer que le grand nombre d'annonces publicitaires ou de nouvelles financières insérées dans le journal Le Progrès d'Athènes, organe officieux - on l'a dit - de la légation de France, obéissent plus nettement à ces critères de propagande économique et mériteraient d'être examinés de plus près. Ces journaux, enfin, apportent aussi un témoignage précieux sur les activités économiques et commerciales des villes où ils paraissaient ainsi que sur les pratiques sociales en vigueur.

\section{Pour conclure}

Au lendemain de la Seconde Guerre mondiale commence le déclin progressif de la presse francophone hellénique. Si un journal, Le Messager d'Athènes, et la revue L'Hellénisme contemporain sont à peu près les seules publications en langue française qui continuent de paraître en Grèce, les journalistes grecs auront de nouveau recours à la langue française à deux moments cruciaux de l'histoire récente du monde grec : celui de la lutte pour libération de Chypre du joug colonial britannique, et durant la dictature imposée à la Grèce par la junte des colonels. En 1957-1958, Le Héros, publié à Athènes, est l'organe de combat pour la libération de Chypre. Il emprunte la langue française, en parallèle avec le grec et l'anglais, pour mobiliser l'opinion européenne. Et pendant la dictature des colonels, les réfugiés politiques grecs en France lancent de nouveau une publication - polygraphiée cette fois - visant à faire connaître autant la lutte menée par les forces démocratiques que les atrocités du régime ${ }^{31}$. La dernière tentative de création d'un journal de langue française en Grèce remonte à 1997. La Tribune bellénique, diffusée à 2000 exemplaires sur tout le territoire, se voulait, selon son éditeur « une héritière sans fortune de ce passé prestigieux ${ }^{32}$ ». Elle s'éteint deux ans plus tard sans laisser de postérité.

30. StaVrinou, 1996, p. 92.

31. SOMÉRITIS, 2008, p. 397.

32. http://www.tv5monde.com/TV5Site/athenes/fr/lavie/afrancais.html. Voir aussi KraEMER, 2001. Il ne m'a pas été possible jusqu’à ce jour de trouver des exemplaires de ce journal. 
Ce premier état des lieux de la presse hellénique de langue française, encore lacunaire et incomplet à ce stade de la recherche, suscite de multiples interrogations auxquelles j'espère pouvoir répondre dans la suite de ma recherche. Néanmoins, ce que l'on peut déjà affirmer avec certitude, c'est que cette presse a été investie d'une double mission, à la fois civilisatrice et propagandiste. Car c'est par l'intermédiaire de ces journaux rédigés en français - entièrement ou partiellement - que la Grèce peut faire entendre sa voix en Europe et informer l'opinion publique européenne sur les faits politiques et les problèmes multiples qu'affronte le pays. Il s'agit donc avant tout de véhiculer un message politique et de donner une tribune aux revendications nationales.

On constate aussi que les journalistes et publicistes qui ont décidé de publier en français avaient pour objectif avoué et avéré de nouer un dialogue avec l'Europe à des moments cruciaux pour l'avenir. Leur détermination entendait faire sortir la Grèce de son isolement et lui assurer de bonnes alliances susceptibles de promouvoir ses revendications. À cette fin, ils ont aussi voulu élaborer une nouvelle image du pays et ont mis en avant la culture.

Cette presse en partie ou entièrement francophone, qui occupe une place particulière dans le paysage journalistique grec, mérite que l'on s'y intéresse dans le cadre de l'historiographie de la presse nationale au même titre que les journaux en langue grecque. En effet, la presse hellénique en langue française ou bilingue transcrit l'évolution politique du pays, participe à la lutte pour les questions nationales - parfois même férocement -, et se fait le reflet des réalités historiques et sociales. Elle participe à l'élaboration de l'idéologie nationale et devient la voix de la Grèce en France et en Europe.

Ces premiers constats établis, on peut dire également ce qui reste à faire car la recherche, on l'a dit, est encore ouverte. Il convient de procéder à l'analyse des périodiques eux-mêmes, qui est pour l'instant limitée et insuffisamment reliée à leur contexte historique et culturel. Il faut aussi étudier le rôle de ces journalistes, médiateurs culturels ou agents d'intérêts étrangers, autant au plan individuel qu'en tant que catégorie socioprofessionnelle distincte. À terme, c'est la reconstitution des réseaux mis en place autour de la presse de langue française qui est visée. En effet, outre le contenu même de leurs publications, l'étude de leur cercle éditorial particulier et de ses stratégies apparaît comme une étape prometteuse pour une meilleure compréhension des réseaux qui se créent entre la métropole et l'émigration, entre la France et la Grèce, deux pays qui demeurent, aujourd'hui encore, étroitement liés, en dépit de l'absence d'une telle presse à présent. 
CAHIERS BALKANIQUES

48

La presse allophone dans les Balkans

\section{Bibliographie}

\section{Monographies}

Christopoulos Panayotis, 1994, Newspapers in the Greek chamber of Deputies Library (1789-1970). Descriptive catalogue, Library of the Greek Chamber of Deputies - Center of Neohellenic Research/NHRF, Athens.

Eglezou Georgia, 2009, The Greek media in World War I and its aftermath: the Athenian press and the Asia Minor crisis. Tauris Academic Studies, London, 288 p.

Droulia Loukia [Dporaia $\Lambda$ ovki $]$ \& Koutsopanagou Yioula

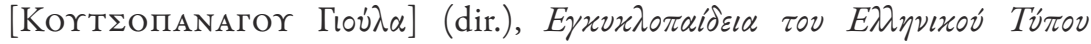
1784-1974 [Encyclopédie de la presse hellénique], 2008, 4 vol. EӨvıкó 'I $\rho \nu \mu \alpha$

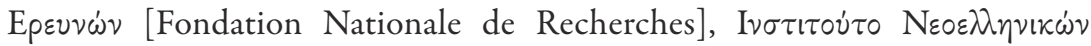

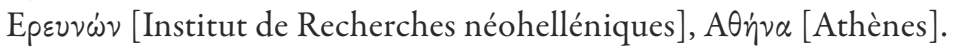

Kitsikis Dimitri, 1963, Propagande et pressions en politique internationale. La Grèce et ses revendications à la conférence de la Paix. PUF, Paris, 539 p.

Kraemer Gilles, 2001, La presse francophone en Méditerranée. Servédit-Maisonneuve \& Larose, Paris.

Papadopoulou Despina, 2004, Les Grecs à Paris à la fin du xix siécle : la construction d'une communauté migrante. Thèse de doctorat non publiée, EHESS, Paris, 372 p.

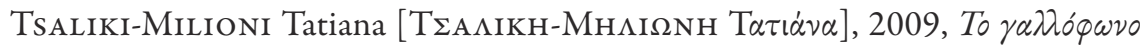

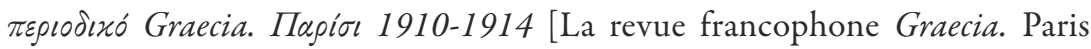

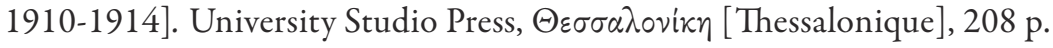

\section{Articles et participations à des ouvrages}

Arnoux-Farnoux Lucile, 2015, « Lettres grecques en France - Deux revues néo-hellénistes : Libre (1924-1936) et les Études Néo-belléniques (1968-1976) »,

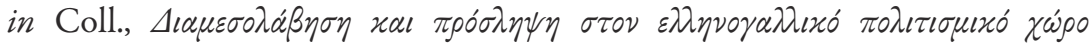

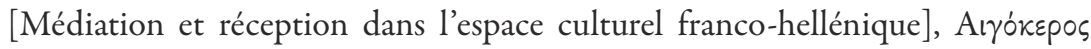

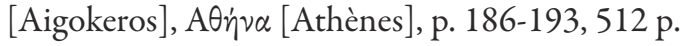


Arnoux-Farnoux Lucile, « La presse francophone grecque et la promotion de la culture néohellénique. Le cas du Monde Hellénique (14 avril 1906-25 avril 1910) », in Empereur Jean-Yves \& Martellière Marie-Delphine (dir.), 2017, Pressesallophones de Méditerranée. Centre d'Études alexandrines, Alexandrie, p. 265-273, 369 p.

BRUneaU Michel, 1996, « Une immigration dans la longue durée : la diaspora grecque en France », Espace, populations, sociétés, 1996-2- 3. Immigrés et enfants d'immigrés. p. 485-495. URL : http://www.persee.fr/doc/ espos_0755-7809_1996_num_14_2_1775 (consulté le 19/04/2017).

Flitouris Lampros, 2017, « La presse francophone de Salonique (XIX ${ }^{e}$ siècle-1921)», inEmpereurJean-Yves\&Martellière Marie-Delphine (dir.), Presses allophones de Méditerranée. Centre d'Études alexandrines, Alexandrie, p. 255-263, 369 p.

Ganiage Jean, 2001, « Les luttes entre chrétiens en Macédoine. Grecs et Serbes contre Bulgares (1904-1908) », Guerres mondiales et conflits contemporains 2001/2, $\mathrm{n}^{\circ}$ 202-203, p. 113-136. URL: https://www.cairn.info/revue-guerres-mondiales-etconflits-contemporains-2001-2-page-113.htm

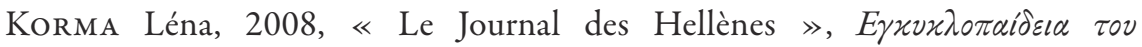

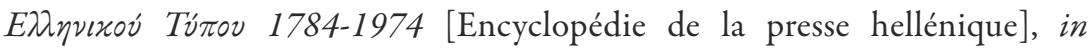
Droulia Loukia [ $\triangle$ porsia $\Lambda$ ouki $\alpha]$ \& Koutsopanagou Yioula

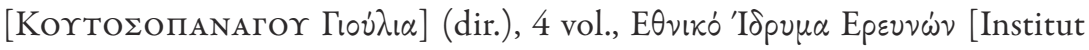

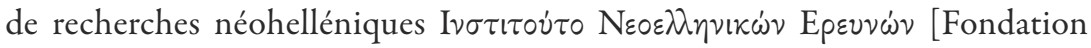
nationale de recherches], $A \theta \dot{\eta} v \alpha$ [Athènes], p. 428-430, 542 p.

LouÉ Thomas, 2005, « Les passeurs culturels au risque des revues », in Cooper-Richet Diana, Mollier Jean-Yves \& Silem Ahmed (dir.). Passeurs culturels dans le monde des médias et de l'édition en Europe ( $\mathrm{xix}^{e}$ et $\mathrm{xx}^{e}$ siècles). Presses de l'ENSIBB, Villeurbanne, 195-208, $352 \mathrm{p}$.

Manitakis, Nicolas, 2000, « Travailleurs immigrés grecs en France, 19161931 : massification et enracinement d'un mouvement migratoire », in Grivaud Gilles (dir.), La Diaspora hellénique en France, Actes du séminaire organisé à l'École française d'Athènes (18 octobre- ${ }^{\text {er }}$ novembre 1995), p. 83-109. 


\section{CAHIERS BALKANIQUES}

50

La presse allophone dans les Balkans

Montant Jean-Claude,1992, « Aspects de la propagande française en Grèce pendant la Première Guerre mondiale », in La France et la Gréce dans la Grande Guerre : actes du colloque tenu en novembre 1989, université de Thessalonique, Thessalonique, p. 61-87.

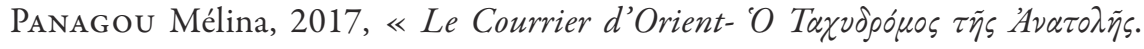
Portrait d'un journal franco-hellénique du début $\mathrm{du} \mathrm{Xx}^{\mathrm{e}}$ siècle », in Empereur Jean-Yves \& Martellik̀re Marie-Delphine (dir.), Presses allophones de Méditerranée. Centre d'Études alexandrines, Alexandrie, p. 275-280.

Provata Despina, 2007, « La Grèce de Juliette Adam : écrits littéraires et vision politique », Revue des études néo-helléniques 3, Daedalus, Paris-Athènes, p. 63-76.

Provata Despina, 2011, « Construction identitaire et enseignement du français en Grèce au XIX ${ }^{\mathrm{e}}$ siècle » in Dimadis Konstantinos (ed.), Identities in the Greek world (from 1204 to the present day). 4th European Congress of Modern Greek Studies, Granada, 9-12 September 2010, vol. 5, European Society of Modern Greek Studies, Athens, p. 281-192, 888 p.

Provata Despina, 2017, « La presse francophone grecque. Revendications nationales et ouverture vers l'Europe », in EMPEREUR Jean-Yves \& Martellière Marie-Delphine(dir.),2017, Presses allophones de Méditerranée. Centre d'Études alexandrines, Alexandrie, p. 281-296.

Soméritis Richardos, 2008, « Athènes-Presse Libre », in Droulia Loukia

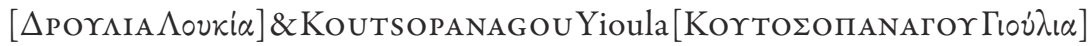

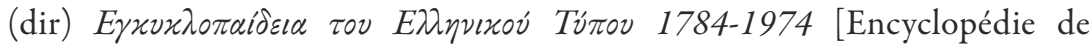

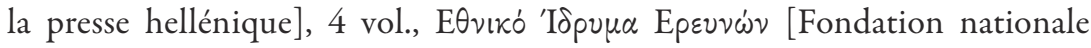

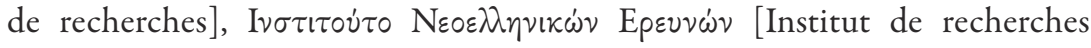
néohelléniques], $\mathrm{A} \theta \dot{\eta} \nu \alpha$ [Athènes], $542 \mathrm{p}$.

Stavrinou Miranda, 1996, «Gustave Fougères, l'École française d'Athènes et la propagande en Grèce durant les années 1917-1918 », Bulletin de correspondance hellénique, vol. 120, livraison 1, 1996, p. 83-99.

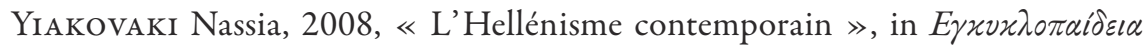

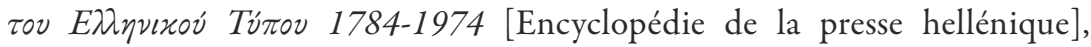


Droulia Loukia [Dporaia $\Lambda$ ouki $\alpha$ ] \& Koutsopanagou Yioula

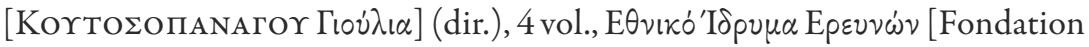

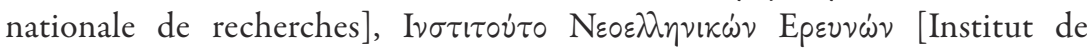

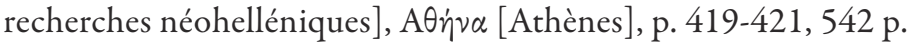

Résumé : cet article est une première approche de l'histoire de la presse francophone grecque de la première moitié $\mathrm{du} \mathrm{xx}^{\mathrm{e}}$ siècle. Il veut dans ce premier temps établir un inventaire aussi raisonné que possible pour cette époque afin d'en dégager les lignes de force qui permettront d'affiner sa périodisation, de dessiner sa typologie et de recenser les initiateurs de cette production éditoriale particulière.

Mots-clefs : Grèce, première moitié $\mathrm{du} \mathrm{Xx}^{\mathrm{e}}$ siècle, presse francophone, médiateurs culturels, transferts culturels

Abstract: This paper is a first attempt to examine the history of the Greek Frenchlanguage press of the first half of the 20th century. At this preliminary stage of my project, the paper makes an inventory of this period and aims to identify its main tendencies in order to refine its periodisation, establish a typology and list the main actors who are responsible for this editorial production.

Keywords: first half of the $20^{\text {th }}$ century, French-speaking press, Greece, cultural mediators, cultural transfers

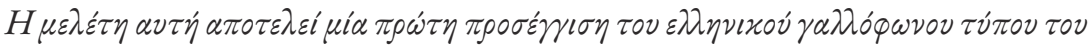

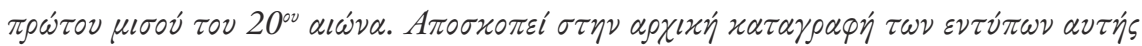

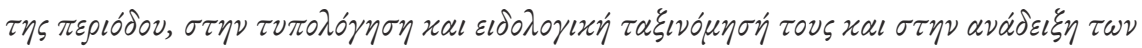

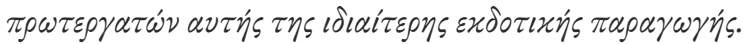

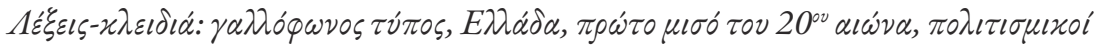

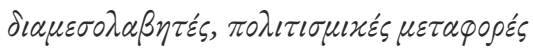

Anahtar kelimeler: 20. yüzyılın ilk yarısı, Fransız basını, temalar, okuyucular, tipoloji, Yunanistan

Клучни зборови: Гриија, првата половина на 20 век, теми, читатели, mипологија, франиускиот печат 